\title{
城市轻轨车辆带摇枕转向架的结构方案 设计和动力学性能
}

周 橙 $^{1}$ 池茂儒 $^{1}$ 梁树林 $^{1}$ 王湘涛 $^{2}$ 周 飞 ${ }^{2}$ 吴兴文 $^{1}$

(1. 西南交通大学牵引动力国家重点实验室 成都 610031;

2. 通号轨道车辆有限公司 长沙 410100)

摘要: 城市轨道交通应用范围越来越广, 除市内运行要求外, 目前还出现了市郊运行(运行速度达到 $120 \mathrm{~km} / \mathrm{h}$ )需求。为解决 目前城市轻轨列车的跨线提速运营能力不足问题, 提出一种跨线提速带摇枕轻轨转向架结构方案, 并对其动力学性能进行了 研究。该方案主要在车体与构架之间增加了摇枕结构, 以实现车体构架摇头运动约束解耦与回转阻尼的非线性。在对转向架 结构分析的基础上, 对于无摇枕转向架与带摇枕转向架构架、摇枕与车体进行受力分析, 并建立 5 模块低地板轻轨列车动力 学模型, 分析两种转向架新轮、磨耗轮状态下的稳定性、平稳性、安全性(包括 $R 350$ 大半径曲线与 $R 50$ 小半径曲线)与回转 阻力系数。结果表明: 由于转向架摇枕带来的回转阻力的非线性特性, 带摇枕转向架的稳定性、平稳性与大、小半径下曲线 通过安全性均优于无摇枕转向架。该转向架既可胜任市郊高速运行的要求(保证 $120 \mathrm{~km} / \mathrm{h}$ 运行), 又能胜任市内小半径曲线安 全通行的要求(安全通过 $R 50$ ), 为跨线提速运营提供了保障。

关键词: 动力学性能; 城市轻轨车辆; 跨线提速; 带摇枕转向架

中图分类号: U270

\section{Structural Design and Dynamics Performance Urban Light Rail Vehicles Bogies with Bolster}

\section{ZHOU Cheng $^{1}$ CHI Maoru ${ }^{1}$ LIANG Shulin ${ }^{1} \quad$ WANG Xiangtao $^{2}$ ZHOU Fei $^{2}$ WU Xingwen ${ }^{1}$}

(1. State Key Laboratory of Traction Power, Southwest Jiaotong University, Chengdu 610031;

2. Tong Hao Railway Vehicles Co., Ltd., Changsha 410100)

\begin{abstract}
With the wider application of urban rail transit, in addition to the operational requirements of the city, there is a demand for suburban operation (running speed up to $120 \mathrm{~km} / \mathrm{h}$ ). In order to solve the problem of speed increase and cross line operation of urban light rail vehicles, a bogie structure design with bolster and studies its dynamic performance is proposed. The scheme mainly adds a bolster structure between the carbody and the frame, and achieves the decoupling of yaw motion constraint and the non-linearity of rotation damping. Through the analysis of the bogie structure, the force condition of the bogie, the bolster and the carbody are studied. The low-floor vehicle train model is established based on the system dynamics method. The stability, ride quality, safety (including the $R 350$ large radius curve and the $R 50$ small radius curve) and the bogie X-factor (bogie rotational resistance coefficient) of two bogies are analyzed. Due to the non-linear characteristics of the rotation damping, the dynamic performance of bogies with bolsters is better than that of bogies without bolster under all operation conditions. The bogie can not only meet the requirements of high-speed operation in suburbs (to ensure $120 \mathrm{~km} / \mathrm{h}$ operation), but also meet the requirements of safety of small radius curve in the city (safely through $R 50$ ), which provides guarantee for cross-line and speed-up operation.
\end{abstract}

Key words: dynamics performance; light rail vehicle; speed increase and cross line operation; bogie with bolster

\section{0 前言}

现代城市轻轨列车起源于 1879 年西门子有轨

* 牵引动力国家重点实验室自主课题资助项目(2018TPL_T04)。20180324 收到初稿, 20180928 收到修改稿
电车, 在 20 世纪初期得到迅猛发展, 并于 20 世纪 70 年代以后迎来复兴。1978 年, 国际公共交通联会 UITP 正式将这种城市轨道交通方式定名为轻轨交 通 ${ }^{[1]}$ 。目前我国在北京、沈阳、大连、长春、南京、 苏州、成都、武汉等 18 个城市均已建有城市低地板 轻轨交通系统 ${ }^{[2]}$ 。现代化轻轨交通具有载客量大、 
环境无污染、运行成本低、方便、快捷、舒适、安 全等特点, 适合作为中等城市的主要交通工具, 也 适合作为大城市中大运量交通工具的辅助, 加强市 区外围与主城区之间的联系或用于外围新城及工业 开发区内部 ${ }^{[3-4]}$ 。

目前的城市轻轨列车基本都在市内运行, 曲线 半径很小 $(R 50)$, 运行速度较低(小于 $80 \mathrm{~km} / \mathrm{h}$ )。为了 适应城市环境运营条件, 现代城市轻轨车辆在发展 过程中出现了与常规车辆区别较大的技术特点。除 弹性车轮、降噪车轮、模块化设计、磁轨制动、超 级电容、燃料电池等新型技术外 ${ }^{[5-7]}$, 列车结构设计 上出现了多种多样的形式。在车辆结构设计上, 出现了单车型式、浮车型式、铰接型式以及单浮 车组合型式等多种编组与铰接方案 ${ }^{[8-10]}$; 在转向架 结构设计上, 出现了传统转向架、单轮对转向架、 独立车轮轮座、自调节车轮轮副、Citadis 耦合转

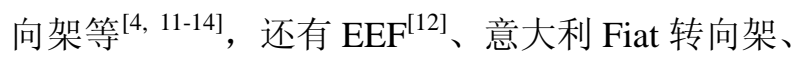
RTX1 转向架 ${ }^{[11]}$ 、径向转向架等特殊结构设计转向 架 ${ }^{[15]}$; 而在轮对导向的设计上, 有小轮径轮对、独 立车轮、踏面设计 ${ }^{[13,16]}$ 、主动导向控制、柔性耦合 轮对 ${ }^{[17]}$ 等方法 $^{[18-20]}$ 。

随着城市交通的发展, 城市轻轨除了市内运行 外, 可能会延伸到市郊(运行速度达到 $120 \mathrm{~km} / \mathrm{h}$ ), 这样就给轻轨车辆转向架提出了更高的技术要求: 既要具备较好的小半径曲线通过能力, 又要具备高 速运行的稳定性; 而目前城市轻轨车辆转向架跨 线提速运行的能力还有所不足。鉴于此, 本文设 计了一种适宜于城市轻轨车辆跨线提速运行的带 摇枕转向架结构方案, 并对其动力学性能开展了 深入研究。

\section{1 转向架方案设计}

列车采用城市轻轨 $100 \%$ 低地板列车常用的 $\mathrm{M}+\mathrm{F}+\mathrm{T}+\mathrm{F}+\mathrm{M}$ 浮车结构型式。 $\mathrm{M}$ 代表动车, $\mathrm{T}$ 代表 拖车, $\mathrm{F}$ 代表无转向架的浮车。浮车下方没有转向 架支撑，通过铰接装置 “挂” 在与其相邻的车体上。 浮车型轻轨列车具有转向架数目少, 成本较低, 车 内空间大, 曲线通过性能较好等特点, 是目前 $100 \%$ 低地板列车的主要形式。Siemens 的 Combino, Bombardier 的 Flexity Outlook 系列, Alstom 的 Citadis 系列、AnsoldoBreda 的 Sirio 系列以及日本的 Jtram 系列 ${ }^{[8]}$ 、国内长客 $100 \%$ 低地板系列、浦镇 $100 \%$ 低 地板系列、通号 100\%低地板等都采用这种型式。 图 1 为 Flexity outlook 的整体视图, 是典型的 5 模 块浮车型式。

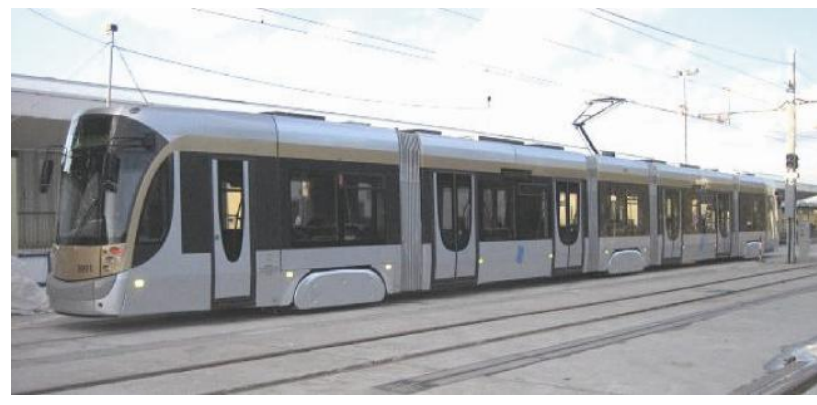

图 1 浮车形式 $100 \%$ 低地板轻轨车 Flexity outlook

以头车动力转向架为例, 分别对常规转向架与 跨线提速带摇枕转向架进行结构说明。

常规转向架结构形式如图 2 所示, 为无摇枕转 向架。转向架采用常规轮对形式走行与导向, 并采 用小轮径车轮以降低地板面高度。为达到紧凑型设 计要求, 轴箱采用内轴箱结构。一系采用钢簧悬挂, 二系采用沙漏簧。电机齿轮箱悬挂在构架上，纵向 进行布置, 采用伞齿轮直角传动进行转矩传递。转 向架与车体采用单拉杆结构实现纵向牵引力与制动 力的传递。每转向架均设有二系横向减振器、二系 垂向减振器、抗侧滚扭杆装置。

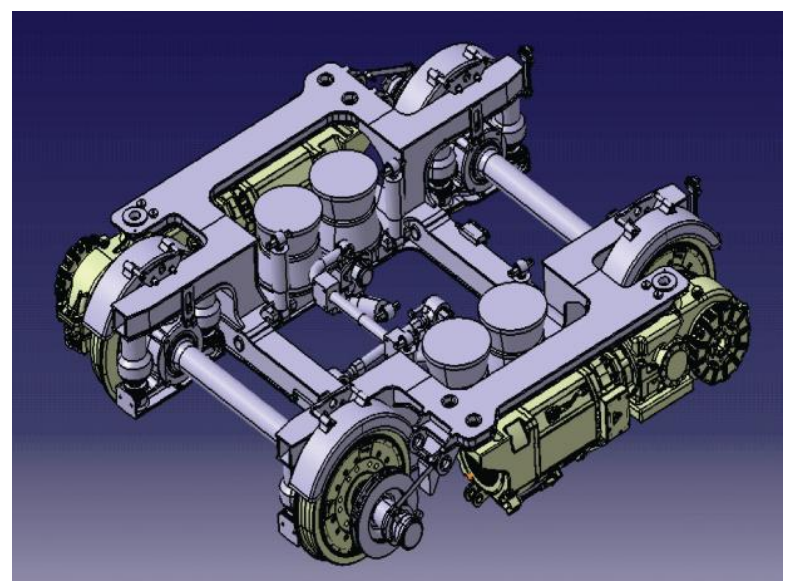

图 2 无摇枕转向架示意图

新设计的跨线提速运行转向架如图 3 所示, 为 带摇枕转向架。带摇枕转向架结构在无摇枕转向架

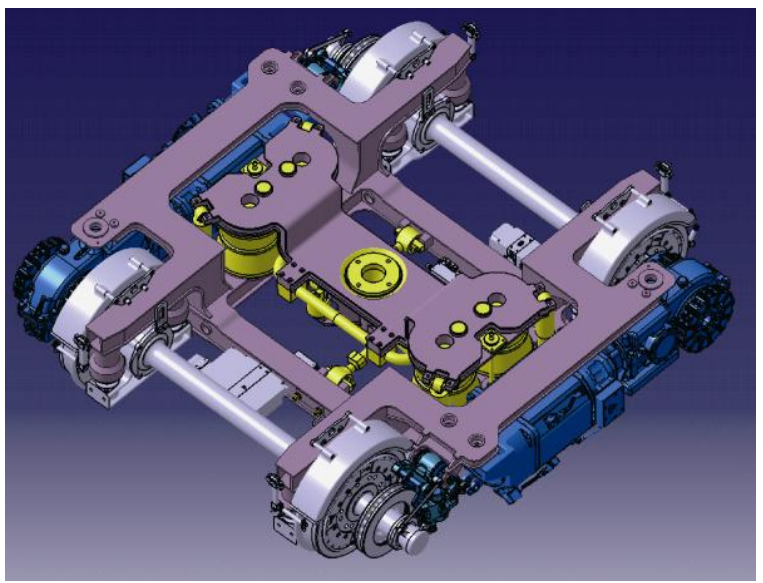

图 3 带摇枕转向架示意图 
二系沙漏簧基础上增加了摇枕机构。摇枕与车体之 间设有旁承装置，提供垂向支撑和摇头阻尼。电机 齿轮箱同样采用纵向布置和伞齿轮直角传动。摇枕 与构架之间采用斜对称布置双牵引拉杆进行纵向力 传递。每转向架同样均设有二系横向减振器、二系 垂向减振器、抗侧滚扭杆装置 ${ }^{[21-22] 。}$

带摇枕转向架与无摇枕转向架主要区别在于摇 枕结构与旁承结构。摇枕结构起到释放车体与转向 架之间的摇头动力学约束作用; 旁承结构不仅支撑 车体全部重量, 还可以提供一定的摩擦力。当车体 转向架相对摇头较小时, 带摇枕转向架与无摇枕转 向架相似; 而当车体转向架相对摇头较大时, 二系 变形固定, 车体摇头得到释放, 旁承摩擦力在摇头 过程中起到阻尼作用。在这样的设计下, 带摇枕转 向架既可以保证高速下的稳定性, 也具备了较好的 小半径曲线通过能力, 具备市内、城郊跨线提速运 行潜力。

\section{2 动力学模型建立}

将无摇枕转向架与带摇枕转向架放入 5 模块列 车模型中进行对比。模型中车体、构架、轮对均考 虑伸缩、横移、浮沉、侧滚、点头和摇头六个自由 度, 摇枕考虑浮沉、摇头、侧滚 3 个自由度。车间 铰接分为全约束的固定铰、约束纵横向运动的转动 铰与只约束横向自由度的自由铰三种, 固定铰为下 铰, 转动铰与自由铰为上铰, 上下铰接共同连接了 动车、浮车与拖车。轮对采用传统轮对, 包含各方 向共 6 个自由度。无摇枕转向架列车模型总自由度 为 84 , 带摇枕转向架列车模型总自由度为 93 。轮轨 力法向力采用 Hertz 接触算法, 切向力采用用 Kalker 简化理论进行计算。对无摇枕转向架与带摇枕转向 架进行受力分析。

\section{1 无摇枕转向架动力学模型}

无摇枕转向架车辆构架受力如图 4 所示 ${ }^{[16,23-25]}$ 。 构架垂向、横向、侧滚、点头和摇头的运动微分方 程为

$$
\begin{gathered}
M_{t} \ddot{Z}_{t}=F_{z t L 1}-F_{z f L 1}-F_{z f L 2}+F_{z t R 1}-F_{z f R 1}-F_{z f R 2}+ \\
M_{t} g+F_{C s z L 1}+F_{C s z L 2}+F_{C s z R 1}+F_{C s z R 2} \\
M_{t} \ddot{Y}_{t}=F_{y f L 1}+F_{y f R 1}-F_{y t L 1}+F_{y f R 1}+F_{y f R 2}-F_{y t R 1}- \\
F_{C s y L 1}-F_{C s y R 1} \\
I_{t x} \ddot{\phi}_{t}=-\left(F_{y f L 1}+F_{y f R 1}+F_{y f L 2}+F_{y f R 2}\right) H_{t w}+\left(F_{z f L 1}+\right. \\
\left.F_{z f L 2}-F_{z f R 1}-F_{z f R 2}\right) d_{w}+\left(F_{z t R 1}-F_{z t L 1}\right) d_{s}-\left(F_{y t L 1}+\right. \\
\left.F_{y t R 1}\right) H_{B t}+\left(F_{C s y L 1}+F_{C s y R 1}\right) H_{C s y t}+\left(F_{C s z R 1}+F_{C s z R 2}-\right.
\end{gathered}
$$

$$
\begin{gathered}
\left.F_{C s z L 1}-F_{C s z L 2}\right) d_{C s z t}+M_{R 1} \\
I_{t y} \ddot{\beta}_{t}=\left(F_{z f L 1}+F_{z f R 1}-F_{z f L 2}-F_{z f R 2}\right) l_{t}- \\
\left(F_{x f L 1}+F_{x f R 1}+F_{x f L 2}+F_{x f R 2}\right) H_{t w}+F_{d r a w 1} H_{D t}- \\
\left(F_{x t L 1}+F_{x t R 1}\right) H_{B t}-\left(F_{C s z R 1}-F_{C s z R 2}+F_{C s Z L 1}-\right. \\
\left.F_{C s Z L 2}\right) l_{C s z t} \\
I_{t z} \ddot{\psi}_{t}=\left(F_{y f L 1}+F_{y f R 1}-F_{y f L 2}-F_{y f R 2}\right) l_{t}+\left(F_{x f R 1}+\right. \\
\left.F_{x f R 2}-F_{x f L 1}-F_{x f L 2}\right) d_{w}+\left(F_{x t L 1}-F_{x t R 1}\right) d_{s}
\end{gathered}
$$

式中, $g$ 为重力加速度; $M_{t}$ 为构架质量; $I_{t x}$ 为构架 侧滚运动惯量, $I_{t y}$ 为构架点头运动惯量, $I_{t z}$ 为构架 摇头运动惯量; $H_{B t}$ 二系悬挂距构架质心高度, $H_{t w}$ 构架质心距轮对质心高度， $H_{C s y t}$ 构架质心距二系横 向减振器高度, $H_{D t}$ 构架质心距牵引拉杆连接点高 度; $d_{s}$ 二系悬挂横向跨距之半; $d_{w}$ 一系悬挂横向跨 距之半, $d_{C s z t}$ 二系垂向减振器横向跨距之半; $l_{t}$ 为轴 距之半, $l_{C s z t}$ 二系垂向减振器纵向跨距之半; $F_{x t j i}, F_{y t j i}$ $F_{z t i i}(i=1, j=L, R)$ 二系簧在 $x, y, z$ 方向的力, $F_{x f j i}, F_{y j j i}$, $F_{z f j i}(i=1,2, j=L, R)$ 一系簧在 $x, y, z$ 方向的力, $F_{C s y j i}$, $F_{C s z j i}(i=1,2, j=L, R)$ 为横向、垂向减振器阻尼力, $F_{\text {draw } j i}(i=1,2, j=L, R)$ 为牵引拉杆纵向力, $M_{R}$ 为抗侧 滚扭杆提供的抗侧滚力矩。

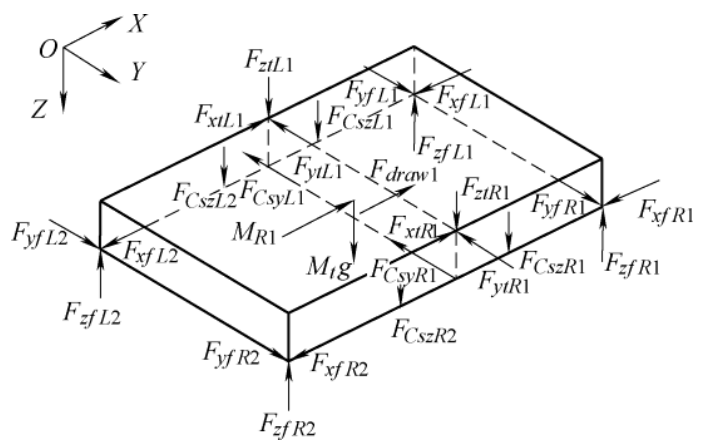

图 4 无摇枕转向架车辆构架受力图

无摇枕转向架车辆头车车体受力如图 5 所示。 车体垂向、横向、侧滚、点头和摇头的运动微分方 程为

$$
\begin{gathered}
M_{c} \ddot{Z}_{c}=-F_{z t L 1}-F_{z t R 1}+M_{c} g-F_{C s Z L 1}- \\
F_{C s z R 1}-F_{C s L L 2}-F_{C s z R 2}-F_{z c d o w n 1} \\
M_{c} \ddot{Y}_{c}=F_{y t L 1}+F_{y t R 1}+F_{C s y L 1}+F_{C s y R 1}+ \\
F_{y c d o w n 1}+F_{y c u p 1} \\
I_{c x} \ddot{\phi}_{c}=-\left(F_{y t L 1}+F_{y t R 1}\right) H_{c B}-M_{R 1}+\left(F_{z t L 1}-\right. \\
\left.F_{z t R 1}\right) d_{s}-\left(F_{C s y L 1}+F_{C s y R 1}\right) H_{C s y c}+\left(F_{C s z L 1}+F_{C s z L 2}-\right. \\
\left.F_{C s z R 1}-F_{C s z R 2}\right) d_{C s z t}-F_{y c d o w n 1} H_{c d o w n}+F_{y c u p 1} H_{c u p} \\
I_{c y} \ddot{\beta}_{c}=\left(F_{z t L 1}+F_{z t R 1}\right) l_{c}-\left(F_{x t L 1}+F_{x t R 1}\right) H_{c B}+
\end{gathered}
$$




$$
\begin{gathered}
\left(F_{C s z R 1}+F_{C s z L 1}\right) l_{C s z c 1}+\left(F_{C s z R 2}+F_{C s L L 2}\right) l_{C s z c 2}- \\
F_{d r a w 1} H_{c D}+F_{x c u p 1} H_{c u p}-F_{x c d w o n 1} H_{c d o w n}+ \\
F_{z c d o w n} l_{c j o \text { int }} \\
I_{c z} \ddot{\psi}_{c}=\left(F_{y t L 1}+F_{y t R 1}+F_{C s y L 1}+F_{C s y R 1}\right) l_{c}+\left(F_{x t R 1}-\right. \\
\left.F_{x t L 1}\right) d_{s}+\left(F_{y c d o w n 1}+F_{y c u p 1}\right) l_{c j o \mathrm{int}}
\end{gathered}
$$

式中, $M_{c}$ 为车体质量; $I_{c x}$ 为车体侧滚运动惯量, $I_{c y}$ 为车体点头运动惯量, $I_{c z}$ 为车体摇头运动惯量; $H_{c B}$ 二系悬挂距车体质心高度, $H_{C s y c}$ 车体质心距二系横 向减振器高度, $H_{c D}$ 车体质心距牵引拉杆连接点高 度, $H_{\text {cup }}$ 车体质心距上铰连接点高度, $H_{c \text { down }}$ 车体质 心距下铰连接点高度; $l_{c}$ 为转向架中心到车体质心 纵向距离, $l_{C s z c i}(i=1,2)$ 二系垂向减振器距车体质心 纵向距离, $l_{\text {joint }}$ 车辆铰接点距车体质心纵向距离; $F_{x c j i}, F_{y c j i}, F_{z c j i}(i=1, j=u p$, down)车间铰接在 $x, y, z$ 方向 的力。

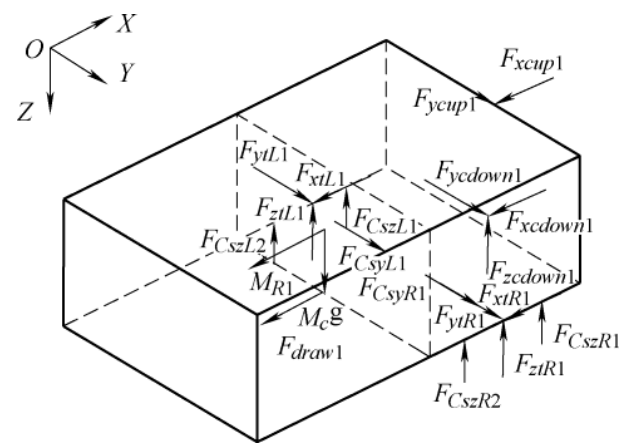

图 5 无摇枕转向架车辆车体受力图

\section{2 带摇枕转向架动力学模型}

带摇枕转向架构架动力学微分方程为与无摇枕 转向架基本相同。

带摇枕转向架车辆摇枕受力如图 6 所示。摇枕 只考虑垂向、侧滚、摇头 3 个自由度, 其他自由度 与车体进行约束, 垂向、侧滚、摇头对应的运动微 分方程为

$$
\begin{gathered}
M_{b o} \ddot{Z}_{b o}=-F_{z t L 1}-F_{z t R 1}+F_{z b o L 1}+F_{z b o R 1}+ \\
M_{b o} g-F_{C s L 1}-F_{C s z R 1}-F_{C s z L 2}-F_{C s z R 2} \\
I_{b o x} \ddot{\phi}_{b o}=-\left(F_{y t L 1}+F_{y t R 1}\right) H_{b o B}+\left(F_{z t L 1}-\right. \\
\left.F_{z t R 1}\right) d_{s}-\left(F_{y b o L 1}+F_{y b o R 1}\right) H_{b o p}+\left(F_{z b o R 1}-F_{z b o L 1}\right) d_{p}- \\
M_{R 1}-\left(F_{C s y L 1}+F_{C s y R 1}\right) H_{C s y b o}+\left(F_{C s z L 1}+F_{C s z L 2}-\right. \\
\left.F_{C s z R 1}-F_{C s z R 2}\right) d_{C s z t} \\
I_{b o z} \ddot{\psi}_{b o}=\left(F_{x t R 1}-F_{x t L 1}\right) d_{s}-\left(F_{x b o R 1}-\right. \\
\left.F_{x b o L 1}\right) d_{p}+\left(F_{d r a w 1}-F_{d r a w 2}\right) d_{d}
\end{gathered}
$$

式中, $M_{b o}$ 为摇枕质量; $I_{c x}$ 为摇枕侧滚运动惯量, $I_{c y}$ 为摇枕点头运动惯量, $I_{c z}$ 为摇枕摇头运动惯量;
$H_{b o B}$ 二系悬挂距摇枕质心高度, $H_{b o p}$ 旁承接触面距 摇枕质心高度, $H_{C s y b o}$ 摇枕质心距二系横向减振器高 度; $d_{p}$ 旁承横向跨距之半; $d_{d}$ 牵引拉杆横向跨距之 半; $F_{x b o j i}, F_{y b o j i}, F_{z b o j i}(i=1, j=L, R)$ 旁承接触在 $x, y, z$ 方 向的力。

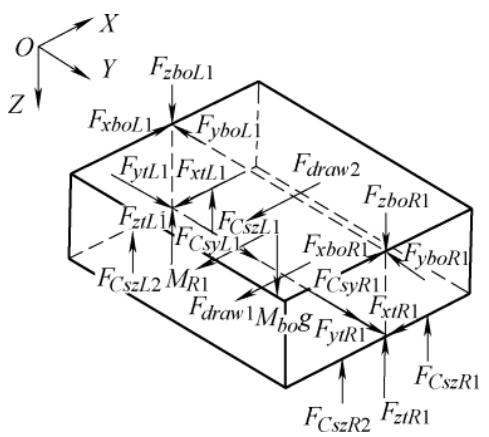

图 6 带摇枕转向架车辆摇枕受力图

带摇枕转向架车辆头车车体受力如图 7 所示。 车体垂向、横向、侧滚、点头和摇头的运动微分方 程为

$$
\begin{gathered}
M_{c} \ddot{Z}_{c}=-F_{z b o L 1}-F_{z b o R 1}+M_{c} g-F_{z c d o w n 1} \\
M_{c} \ddot{Y}_{c}=F_{y b o L 1}+F_{y b o R 1}+F_{y c d o w n 1}+F_{y c u p 1} \\
I_{c x} \ddot{\phi}_{c}=-\left(F_{y b o L 1}+F_{y b o R 1}\right) H_{c b o}+\left(F_{z b o L 1}-\right. \\
\left.F_{z b o R 1}\right) d_{p}-F_{y c d o w n 1} H_{c d o w n}+F_{y c u p 1} H_{c u p} \\
I_{c y} \ddot{\beta}_{c}=\left(F_{z b o L 1}+F_{z b o R 1}\right) l_{c}-\left(F_{x b o L 1}+\right. \\
\left.F_{x b o R 1}\right) H_{c b o}+F_{x c u p 1} H_{c u p}-F_{x c d w o n 1} H_{c d o w n}+ \\
F_{z c d o w n} l_{c j o \text { int }} \\
I_{c z} \ddot{\psi}_{c}=\left(F_{y b o L 1}+F_{y b o R 1}\right) l_{c}+\left(F_{x b o R 1}-F_{x b o L 1}\right) d_{p}+ \\
\left(F_{y c d o w n 1}+F_{y c u p 1}\right) l_{c j o \text { int }}
\end{gathered}
$$

式中, $H_{c b o}$ 为旁承接触面距车体质心高度。

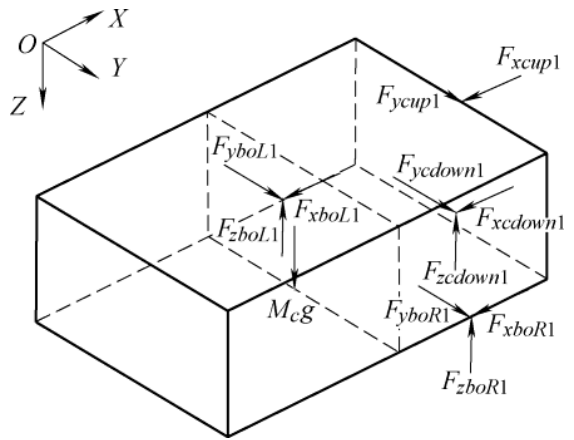

图 7 带摇枕转向架车辆车体受力图

\section{3 车辆动力学性能分析}

取城市低地板轻轨列车的稳定性、平稳性、安 全性和回转阻力系数进行计算, 对比无摇枕转向架 
与带摇枕转向架动力学性能表现, 分析所设计转向 架在跨线提速方面的优势。各性能均采用新轮与磨 耗轮状态进行计算, 相关轮轨接触关系如图 8 所示, 磨耗轮为实测城市轻轨车辆磨耗到限踏面。轨道激 励采用美国五级谱。稳定性与平稳性计算采用直线 工况, 安全性计算采用 $R 350$ 大半径曲线与 $R 50$ 小 半径曲线两种工况。

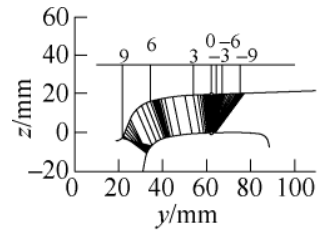

(a) 新轮接触关系

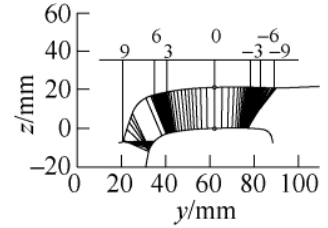

(b) 磨耗轮接触关系

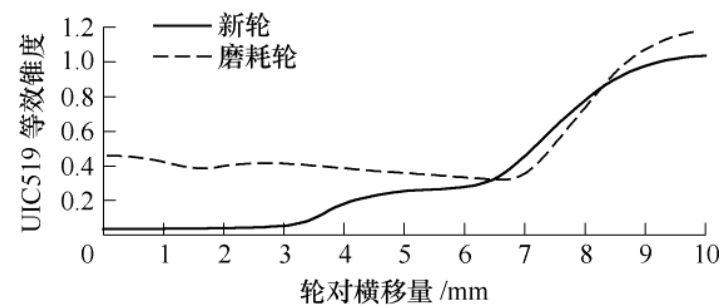

(c) 新轮、磨耗轮等效雉度

图 8 无摇枕转向架与带摇枕转向架稳定性对比

\section{1 稳定性对比}

稳定性采用初始轮对横移量加初始激扰, 观察 轮对收玫情况方法进行计算。对比无摇枕转向架和 带摇枕转向架下新轮、磨耗轮的临界速度与分岔图, 如表 1 与图 9 所示。可以得到如下结论。

\section{表 1 无摇枕转向架与带摇枕转向架临界速度}

\begin{tabular}{ccc}
\hline 转向架形式 & 新轮临界速度 $/(\mathrm{km} / \mathrm{h})$ & 磨耗轮临界速度 $/(\mathrm{km} / \mathrm{h})$ \\
\hline 无摇枕转向架 & 142 & 102 \\
带摇枕转向架 & 186 & 155 \\
\hline
\end{tabular}

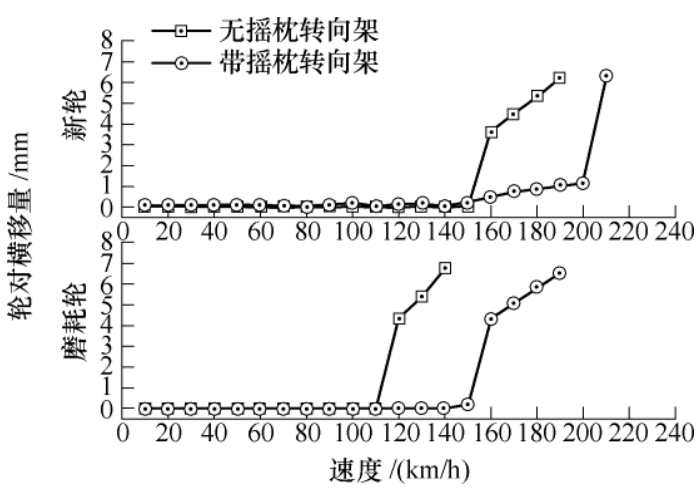

图 9 无摇枕转向架与带摇枕转向架稳定性对比

(1) 带摇枕转向架两种状态下的临界速度均比 无摇枕转向架高。带摇枕转向架旁承通过摩擦力提 供了额外的回转阻尼, 相对于无摇枕转向架稳定性 较好, 能够保证较高临界速度。

(2) 无摇枕转向架与带摇枕转向架、新轮与磨
耗轮分岔形式相同; 踏面变化、转向架结构变化并 未引起一次蛇行现象出现。

(3) 稳定性考虑 $25 \%$ 裕量, 无摇枕转向架只能 满足 $80 \mathrm{~km} / \mathrm{h}$ 的运营要求(计算临界速度 $100 \mathrm{~km} / \mathrm{h}$ ), 带摇枕转向架可以满足 $120 \mathrm{~km} / \mathrm{h}$ 的运营要求(计算 临界速度 $150 \mathrm{~km} / \mathrm{h}$ ), 适合配备于市郊提速城市轻轨 车辆上。

\section{2 平稳性对比}

平稳性计算采用直线工况。对比无摇枕转向架 和带摇枕转向架新轮、磨耗轮状态下的横向平稳性 与垂向平稳性, 如图 10 所示。从图中可以得到如下 结论。

(1) 新轮状态下两种转向架 $20 \sim 120 \mathrm{~km} / \mathrm{h}$ 平稳 性比较接近。

(2) 磨耗轮状态下，低速范围两种转向架平稳 性比较接近; 当速度超过 $80 \mathrm{~km} / \mathrm{h}$ 后, 无摇枕转向 架因为稳定性较差，横向、垂向平稳性均要大于带 摇枕转向架。

(3) 带摇枕转向架低速下平稳性与无摇枕转 向架相当, 高速下平稳性较好, 更适用于市郊提 速运行。

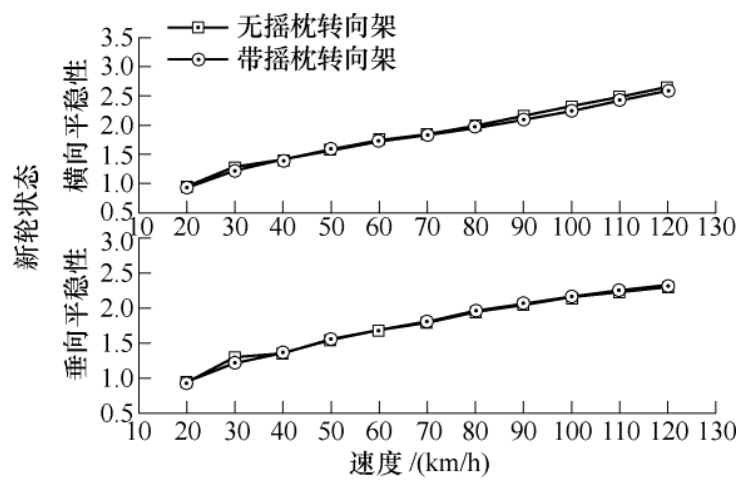

(a) 新轮状态下两种转向架平稳性对比

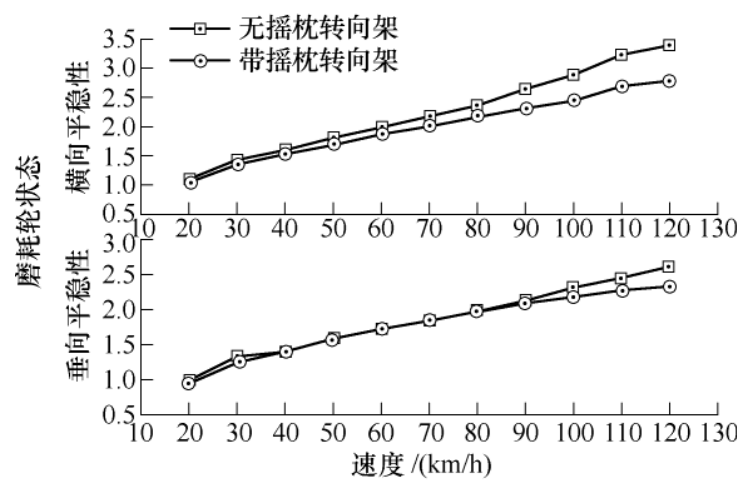

(b) 磨耗轮状态下两种转向架平稳性对比

图 10 无摇枕转向架与带摇枕转向架平稳性对比

\section{3 安全性对比}

安全性计算分为通过 $R 350$ 大半径曲线与 $R 50$ 小半径曲线两种工况。 $R 350$ 限速为 $50 \mathrm{~km} / \mathrm{h}, R 50$ 限速为 $15 \mathrm{~km} / \mathrm{h}$ 。分别对脱轨系数与轮重减载率进行 
分析, 对比两种转向架的曲线通过性能, 如图 11 所示。可以得到如下结论。

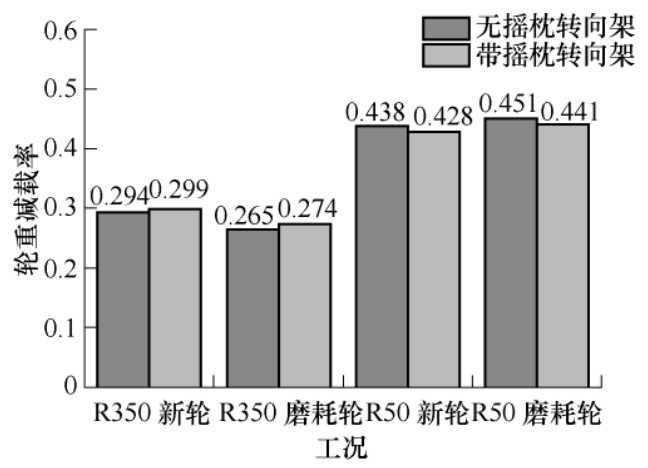

(a) 各状态下两种转向架轮重减载率对比

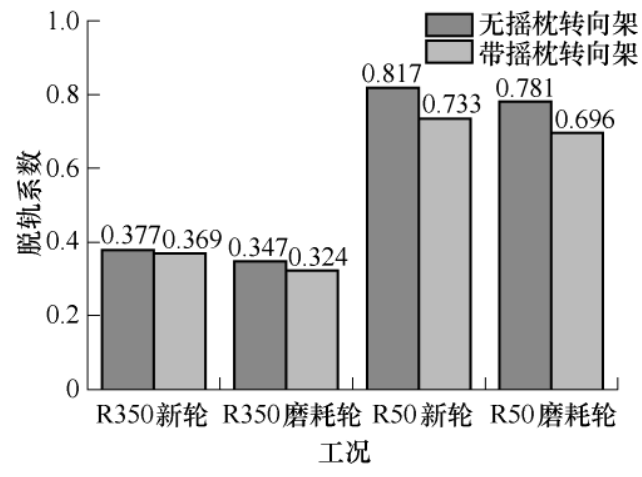

(b) 各状态下两种转向架脱轨系数对比

图 11 无摇枕转向架与带摇枕转向架 $R 350$ 安全性对比

(1) 各工况下两种转向架轮重减载率相近, $R 350$ 曲线下无摇枕转向架略优, $R 50$ 曲线下带摇枕 转向架略优。

(2) 带摇枕转向架 $R 50$ 曲线下脱轨系数要明显 优于无摇枕转向架, $R 350$ 曲线下略优。

(3) 带摇枕转向架曲线通过性能优于无摇枕转 向架, 并且曲线半径越小, 优势越明显, 更适宜于 城市内小半径曲线运营环境。

\section{4 回转阻力系数对比}

根据转向架结构分析可知, 常规无摇枕转向架 与跨线提速带摇枕转向架的区别在于转向架回转能 力差异, 因此取不同摇头角度下回转阻力系数进行 对比, 不考虑阻尼因素。回转阻力系数表示转向架 摇头受到的阻力程度, 定义为

$$
c=T /(Q \times d)
$$

式中, $T$ 为转向架相对车体旋转所需要的扭矩; $Q$ 为转向架车轴在平直轨道上测得的平均轴重; $d$ 为 转向架轴距。

不同角度下回转阻力系数如图 12 所示。从图中 可以得到如下结论。

(1) 无摇枕转向架回转阻力主要由二系悬挂的 纵向刚度提供, 回转阻力系数与旋转角度成正比。

(2) 带摇枕转向架阻力同样由二系悬挂的纵向
刚度提供。当旋转角度较小时, 回转阻力系数与旋 转角度成正比; 当旋转角度较大时, 车体与摇枕出 现相对旋转运动, 旁承摩擦力提供摇头阻尼, 二系 悬挂力不再增大, 回转阻力系数保持不变。

(3) 回转阻力系数说明, 在小回转角度下, 两 种转向架回转能力相似, 带摇枕转向架有额外的回 转摩擦, 可以获得较好的稳定性; 在大回转角度下, 带摇枕转向架回转阻力较小, 摇头约束更小, 在小 半径曲线下同样具有优势。

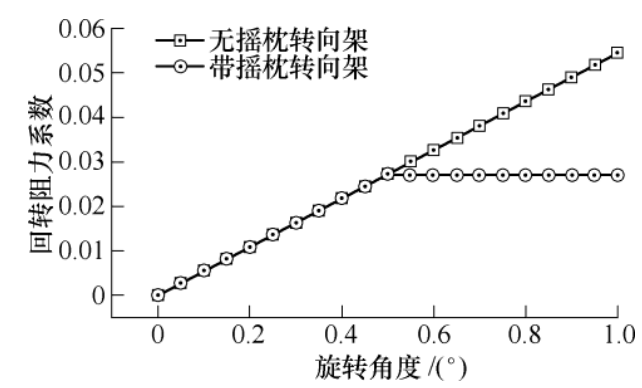

图 12 无摇枕转向架与带摇枕转向架回转阻力系数对比

\section{4 结论}

随着城市轨道交通的发展, 其应用范围越来 越广, 除市内运行要求外, 出现了一定市郊运行(运 行速度达到 $120 \mathrm{~km} / \mathrm{h}$ ) 需求。为解决目前城市轻轨 列车的跨线提速运营能力不足问题, 提出一种跨 线提速带摇枕转向架方案, 并对其动力学性能进 行研究。

(1) 该转向架方案主要在常规城市轻轨转向架 基础上, 在车体与构架之间增加了摇枕机构。摇枕 结构起到释放车体与转向架之间的摇头动力学约束 作用。摇枕与与构架之间通过二系沙漏簧、减振器 等二系悬挂进行连接; 与车体之间通过旁承装置提 供垂向支撑与摇头阻尼, 从而实现回转阻尼的非线 性特性。

(2) 通过对动力学性能与回转阻力系数的分 析, 由于回转阻力的非线性特性, 带摇枕转向架的 稳定性、平稳性与大、小半径下曲线通过安全性均 优于无摇枕转向架。该转向架既可胜任市郊高速运 行的要求(保证 $120 \mathrm{~km} / \mathrm{h}$ 运行), 又能胜任市内小半 径曲线安全通行的要求(安全通过 $R 50$ ), 为跨线提速 运营提供了保障。

\section{参 考 文 献}

[1] 隋悦家. 现代有轨电车及其车辆的发展 [J]. 城市车辆, 2001(5): 36-39.

SUI Yuejia. The Development of modern tram and its 
vehicles [J]. Urban Vehicles, 2001(5): 36-39.

[2] 中国城市轨道交通协会. 城市轨道交通 2017 年度统计 和分析报告[J]. 城市轨道交通，2018(4)：6-25.

China Urban Rail Transit Association. Statistical and analytical report of urban rail transit in 2017[J]. China Metros, 2018(4): 6-25.

[3] 訾海波, 过秀成, 杨洁. 现代有轨电车应用模式及地区 适用性研究[J]. 城市轨道交通研究, 2009, 12(2): 46-49. ZI Haibo, GUO Xiucheng, YANG Jie. Research on the application and adaptability of modern tram car[J]. Urabn Mass Transit, 2009, 12(2): 46-49.

[4] 李䒾, 张丽平, 黄运华. 城市轻轨车辆发展及其应用前 景 $[\mathrm{J}]$. 西南交通大学学报, 2002(2): 111-116.

LI Fu, ZHANG Liping, HUANG Yunhua. The development and prospect of application of light rail vehicles[J]. Journal of Southwest Jiaotong University, 2002(2): 111-116.

[5] 张振沝. 城市轨道交通车辆 $[\mathrm{M}]$. 北京: 中国铁道出版 社, 1998.

ZHANG Zhenmiao. Urban rail transit vehicles[M]. Beijing: China Railway Press, 1998.

[6] 杨继斌, 宋鹏云, 张继业, 等. 混合动力现代有轨电车仿 真系统研究 $[J]$. 机械工程学报, 2017，53(18): 175-182. YANG Jibin, SONG Pengyun, ZHANG Jiye, et al. Research on simulation system of hybrid modern tramway[J]. Journal of Mechanical Engineering, 2017, 53(18): 175-182.

[7] 李明, 刘楠, 韩铁礼. 燃料电池有轨电车冷却装置降噪 设计及试验研究 [J]. 机械工程学报, 2017, 53(4): 97-104.

LI Ming, LIU Nan, HAN Tieli. Noise reduction design and experimental research of cooling system of fuel cell tram[J]. Journal of Mechanical Engineering, 2017, 53(4): 97-104.

[8] 王欢. $100 \%$ 低地板轻轨车辆结构型式与导向机理研究 [D]. 成都: 西南交通大学, 2008 .

WANG Huan. Research of structure style and steering principle of $100 \%$ low floor light rail vehicle[D]. Chengdu: Southwest Jiaotong University, 2008.

[9] BOOZ A. Hamilton Inc. Applicability of Low-floor Light Rail Vehicles in North America. TCRP REPORT 2[R]. Washington, DC: Transportation Research Board, 1995.

[10] 邹鹏. 典型国产低地板有轨电车转向架技术特点分析 [J]. 电力机车与城轨车辆, 2018, 41(3): 78-81.

ZOU Peng. Technical characteristics analysis of typical domestic low-floor tramcar bogies[J]. Electric Locomotives \& Mass Transit Vehicles, 2018, 41(3): 78-81.

[11] 陈泽深, 王成国. 铁道车辆动力学与控制 [M]. 北京: 中国铁道出版社, 2004.
CHEN Zeshen, WANG Chengguo. The dynamics and control of railway vehicles[M]. Beijing: China Railway Press, 2004.

[12] FREDERICH F. Dynamics of a bogie with independent wheels[J]. Supplement to Vehicle System Dynamics, 1989, 19: 217-232.

[13] 任利惠. 独立车轮导向技术研究[D]. 上海: 同济大学, 2006.

REN Lihui. The study on method to enhancing steering capability of independently rotating wheels[D]. Shanghai: Tongji University, 2006.

[14] HONDIUS H, 邹婷婷. Citadis-Dualis 型有轨电车[J]. 国 外铁道车辆, $2008,45(3): 18-19$.

HONDIUS H, ZOU Tingting. Citadis-Dualis tram-train[J]. Foreign Rolling Stock, 2008, 45(3): 18-19.

[15] 许明春. 独立旋转车轮转向架导向性能研究[D]. 成都: 西南交通大学, 2010.

XU Mingchun. A study on steering behabiour of the bogie with independently rotating wheels[D]. Chengdu : Southwest Jiaotong University, 2010.

[16] 王文华. 低地板轻轨车辆动力学问题研究[D]. 长春: 吉 林大学, 2015.

WANG Wenhua. Study of low floor light railway vehicle dynamics[D]. Changchun: Jilin University, 2015.

[17] 池茂儒. 耦合轮对车辆动力学性能的研究[D]. 成都: 西 南交通大学, 2003.

CHI Maoru. A study on dynamics performance of the vehicle with coupled wheelset [D]. Chengdu: Southwest Jiaotong University, 2003.

[18] DARTER M I, KUO C M. Applicability of low-floor light rail vehicles in North America[M]. Washington, DC: Transportation Research Board, 1995.

[19] DUKKIPATI R V, NARAYANA SWAMY S, OSMAN M $\mathrm{O}$ M. Independently rotating wheel systems for railway vehicles-a state of the art review[J]. Vehicle System Dynamics, 1992, 21(1): 297-330.

[20] MICHITSUJI Y, SUDA Y. Running performance of power-steering railway bogie with independently rotating wheels[J]. Vehicle System Dynamics，2006，44(Suppl.): 71-82.

[21] 蔡征征, 王湘涛. 一种低地板转向架及 $100 \%$ 低地板有 轨电车, CN206049695U[P/OL]. 2017-03-29.

CAI Zhengzheng, WANG Xiangtao. A low floor bogie and 100\% low floor tram: China, CN206049695U[P/OL]. 2017-03-29.

[22] 周飞, 王湘涛. 一种带摇枕 $100 \%$ 地板有轨电车转向架, CN105857332A[P/OL]. 2016-08-17.

ZHOU Fei, WANG Xiangtao. A 100\% low floor tram bogie with the bolster: China, CN105857332A[P/OL]. 
2016-08-17.

[23] 翟婉明. 车辆-轨道耦合动力学模型 $[\mathrm{M}]$. 北京: 科学出 版社, 2006.

ZHAI Wanming. Vehicle-track coupled dynamics[M]. Beijing: Science Press, 2006.

[24] 吴兴文. 地震条件下车辆脱轨安全性研究[D]. 成都: 西 南交通大学, 2016.

WU Xingwen. Running safety assessment of railway vehicles under the earthquake excitations[D]. Chengdu: Southwest Jiaotong University, 2016.

[25] LING Liang, HAN Jian, XIAO Xinbiao, et al. Dynamic behavior of an embedded rail track coupled with a tram vehicle[J]. Journal of Vibration \& Control, 2015, 23(14): 2355-2372.
作者简介: 周橙, 男, 1991 年出生, 博士研究生。主要研究方向为车辆 系统动力学。

E-mail: 13882192410@163.com

池茂儒(通信作者), 男, 1973 年出生, 博士, 研究员, 博士研究生导师。 主要研究方向为车辆系统动力学。

E-mail: cmr2000@163.com

梁树林, 男, 1967 年出生, 博士, 教授级高级工程师, 硕士研究生导师。 主要研究方向为结构振动疲劳。

E-mail: a_slliang@163.com

王湘涛, 男, 1964 年出生, 硕士, 高级工程师, 通号车辆公司总工程师。 主要研究方向为城市轨道车辆总体设计与转向架技术。

E-mail: wangxt2002@163.com

周飞, 男, 1984 年出生, 中级工程师, 通号车辆公司转向架设计主管。 主要研究方向为转向架总体与悬挂系统设计。

E-mail: 185427120@qq.com

吴兴文, 男, 1988 年出生, 博士。主要研究方向为车辆系统动力学。 E-mail:84971278@163.com 